Md. Jubaer Alam*, Mohammad Rashed Iqbal Faruque, Taya Allen, Sabirin Abdullah, Mohammad Tariqul Islam, Khairul Nizam Abdul Maulud, and Eistiak Ahamed

\title{
Depiction and analysis of a modified theta shaped double negative metamaterial for satellite application
}

https://doi.org/10.1515/phys-2018-0105

Received May 1, 2018; accepted July 23, 2018

\section{Introduction}

Metamaterials are the special type of materials that are usually not available in nature. They are actually engineered materials, which need to embed periodic unit cell for their formation to create naturally unavailable electromagnetic properties. Moreover, these materials have the power to control the electromagnetic wave beams to show their unorthodox characteristics. These unusual features of the metamaterials totally depend on the geometry of the atomic construction. It has been started from the year 1968, when Veselago et al. [1] observed unique properties of materials having negative permittivity $(\epsilon)$ and permeability $(\mu)$. But it was not appreciated until 2000, when Smith et al. [2] fortunately validated a new kind of a material with unconventional properties (both permittivity and permeability were negative), which is called left-handed metamaterial. In case of negativity, it has been categorized as single-negative (either permittivity is negative or permeability is negative), double-negative (both permittivity and permeability are negative). There is also a term called nearzero refractive index metamaterial (NZRI),where the permittivity and permeability of a material become approximately zero on a particular range of frequency. Having these captivating electromagnetic phenomena, necessary applications, like SAR reduction [3, 4], super lenses, antenna design $[5,6]$, filters $[7,8]$, invisibility cloaking $[9,10]$, solar cell [11], electromagnetic absorber, and electromag-

Mohammad Rashed Iqbal Faruque, Taya Allen, Sabirin Abdullah, Eistiak Ahamed: Space Science Centre (ANGKASA), Institute of Climate Change (IPI), Universiti Kebangsaan Malaysia, 43600 Bangi, Selangor, Malaysia Mohammad Tariqul Islam: Centre of Advanced Electronic and Communication Engineering, Universiti Kebangsaan Malaysia, 43600 Bangi, Selangor, Malaysia

Khairul Nizam Abdul Maulud: Earth Observation Center (EOC), Institute of Climate Change (IPI), Universiti Kebangsaan Malaysia, 43600 Bangi, Selangor, Malaysia
*Corresponding Author: Md. Jubaer Alam: Space Science Centre (ANGKASA), Institute of Climate Change (IPI), Universiti Kebangsaan Malaysia, 43600 Bangi, Selangor, Malaysia, E-mail: jubaer.alam@iubat.edu 
netic band gaps etc. can be employed by metamaterials. In some cases, intrinsic negative permittivity is found. But it is really difficult to find the negative permeability with a natural medium. Even artificial structures can hardly obtain the negative permeability. Concurrently, it is really difficult to get the negative refractive indices. Currently, multi-band metamaterial absorbers have become an auspicious application in the detection of explosives, even in bolometers and thermal detectors. Moreover, a very few studies have been made in designing this type of materials [12-14]. Different alphabetic shapes have become popular for particular operations [15]; like, Benosman et al. [16] introduced a double S-shaped metamaterial that showed negative values of $\eta$ from 15.67 to $17.43 \mathrm{GHz}$. Mallik et al. proposed various U-shaped rectangular array structures left-handed aspect at approximately 5, 6 and 11GHz. A Vshaped metamaterial was presented by Ekmekci et al. - the architecture showed double-negative characteristic. Zhou et al. [17] designed an S-shaped $15 \times 15 \mathrm{~mm}^{2}$ chiral metamaterial for X-and Ku-band application. Though the EMR was not higher than 4. For the purpose of application on $\mathrm{S}$ and $\mathrm{C}$ bands, Alamet al. [18] design H-shaped DNG for different unit cells and array sizes.

A metamaterial unit cell of modified theta-shaped has been proposed in this paper. The structure covers multiple bands (L, C, X, and $\mathrm{Ku}$ ) of frequencies for the transmission coefficient. And for effective parameters, it covers the $\mathrm{X}$ and Ku bands with double negative characteristic.

\section{Cell design}

The diagram of the prospective modified Theta-shaped unit cell composition is itemized in Figure 1. Each unit cell comprises with $9 \mathrm{~mm}$ in length and $9 \mathrm{~mm}$ in width. All elements have the thickness of $0.35 \mathrm{~mm}$. Each Thetashaped split resonator has the width of $0.5 \mathrm{~mm}$ with a same split gap. The outer length of the resonator is $9 \mathrm{~mm}$ where the split of each of the resonators is $0.5 \mathrm{~mm}$. The entire patch (made of copper) is developed on a substrate called FR-4. It has a dielectric constant of $\epsilon \mathrm{r}=4.3$, a dielectric loss-tangent of $\tan \delta \epsilon=0.025$. Sides of the substrate are $\mathrm{L}=\mathrm{W}=9 \mathrm{~mm}$ and the thickness is $\mathrm{t}=1.6 \mathrm{~mm}$. Designed parameters of the proposed metamaterial are enlisted in Table 1.

A prototype array and a unit cell are fabricated for the purpose of measurement. The area of the array is 36 $\times 36 \mathrm{~mm}^{2}$. Two waveguide ports are used to propagate the electromagnetic waves to excite the configuration on two opposite direction of Z-axis. PEC and PMC were used along

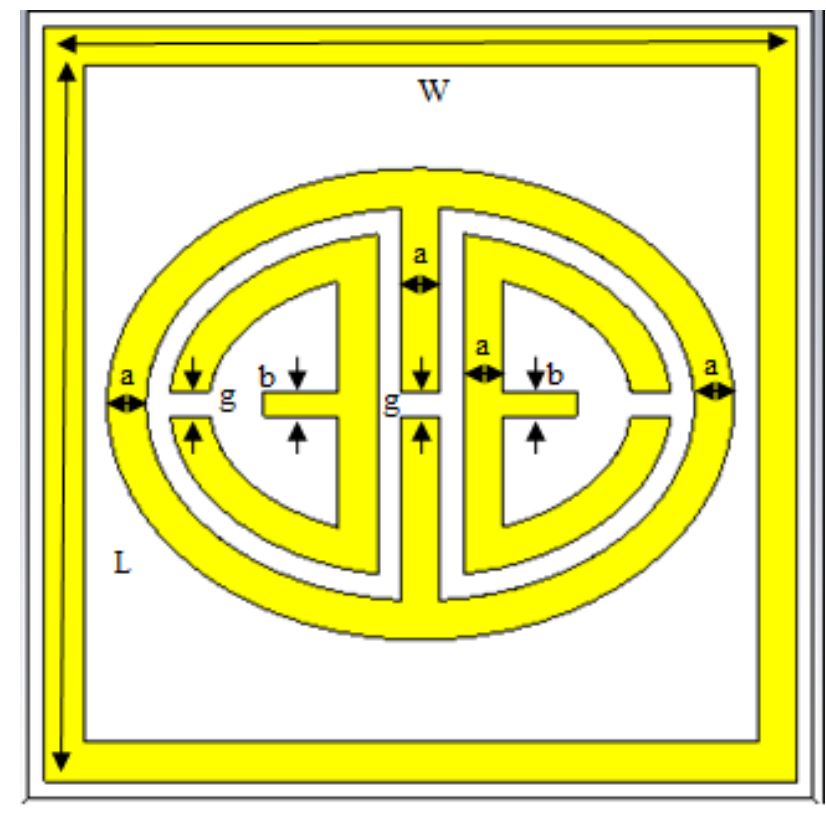

(a)

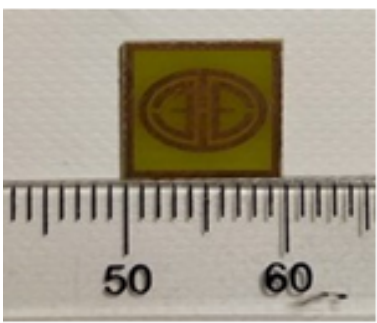

(b)

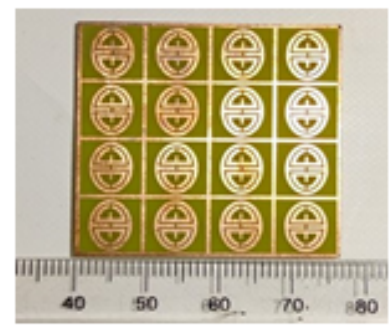

(c)
Figure 1: Metamaterial Unit cell: (a) Proposed geometry; (b) Fabricated geometry; (c) Prototype

the vertical direction of $\mathrm{x}$ and $\mathrm{y}$ axis, respectively. And for the free-space simulation purposes, a frequency domain solver was utilized. Moreover, for the analysis purpose of these configurations, a tetrahedral mesh was used with a flexible mesh. The normalized impedance was $377 \mathrm{Ohms}$ and the system was performed from 1 to $18 \mathrm{GHz}$.

Table 1: Parameters of the unit cell

\begin{tabular}{cc}
\hline Parameters & Dimensions $(\mathrm{mm})$ \\
\hline $\mathrm{L}$ & 9 \\
$\mathrm{~W}$ & 9 \\
$\mathrm{a}$ & 0.5 \\
$\mathrm{~g}$ & 0.3 \\
$\mathrm{~b}$ & 0.3 \\
\hline
\end{tabular}

The area of the prototype was $9 \times 9 \mathrm{~mm}^{2}$, which was fabricated for the purpose of measurements. By settling 


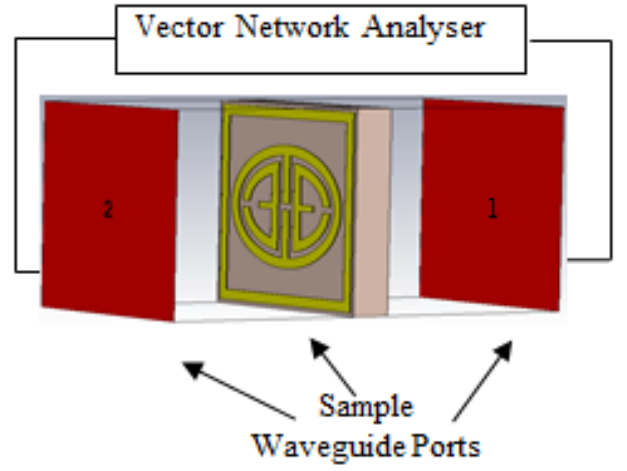

(a)

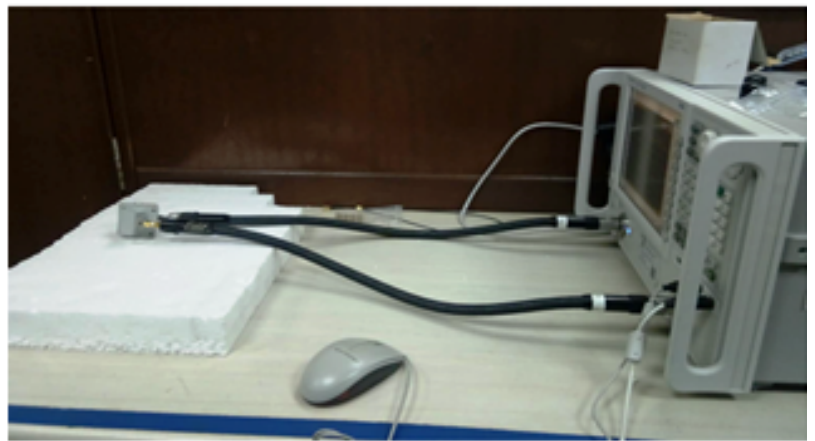

(b)

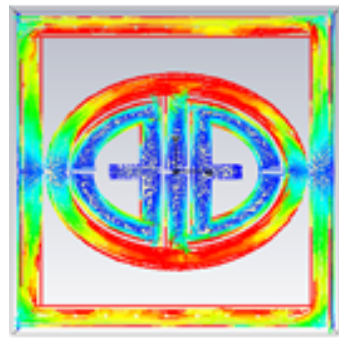

$6.5 \mathrm{GHz}$

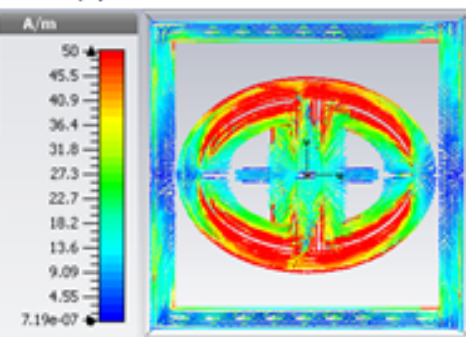

(c)
$13.5 \mathrm{GHz}$
Figure 2: (a) Simulation set up for measuring S parameter; (b) Experimental set up for measuring $S$ parameter; (c) Current distribution of the unit cell at various frequencies

the perspective unit cell in between, the waveguides as per the Figure 2 to actuate the parameters accurately of the metamaterial unit cell. To determine the parameters, we used a vector analyzer commonly known as Agilent N5227A. To calibrate perfectly, an Agilent N4694-60001 was utilized. Figures 2(a) and 2(b) show the simulation and experimental set up of the proposed metamaterial, respectively.

The equation for this type of passive LC circuits of metamaterial structure is

$$
f=\frac{1}{2 \pi \sqrt{L C_{o}}}
$$

Where $\mathrm{L}$ represents cumulative inductance and $\mathrm{C}$ represents cumulative capacitance. Here $\mathrm{C}_{o}$ represents the ca- pacitance required that forms in two adjacent unit cells. Here, metal loops create inductance and splits create capacitance. When the electromagnetic waves applied through the structure, two types of coupling occurred. Electric resonances are produced due to the formation of coupling between gaps and electric field. Magnetic resonances are formed because of loops and magnetic field. Commonly, the total capacitance formed between the gaps is:

$$
C=\epsilon_{0} \epsilon_{r} \frac{A}{d}(F)
$$

where $\epsilon_{0}$ is free space permittivity and $\epsilon_{r}$ is relative permittivity. A infers the cross-sectional area of the gap and $d$ refers to the gap length. Hence, the total inductance can be estimated from [19] as:

$$
L_{1}=\mu_{0}\left\{\frac{2 c}{2 w+h}+\frac{\sqrt{(2 w+h)^{2}+l^{2}}}{c}\right\} t
$$

Therefore, the equivalent capacitance will be apparent as:

$$
C_{o} \approx \epsilon_{0} \frac{(2 w+h)}{\pi} \ln \left(\frac{2 c}{a-l}\right)
$$

Where $\mu_{0}$ is $4 \pi \times 10^{-7} \mathrm{H} / \mathrm{m}, \epsilon_{0}$ is $8.854 \times 10^{-12} \mathrm{~F} / \mathrm{m}$, width $w$, height hand length $l$. The resonances of the proposed structure are formed because of several series and parallel inductances and capacitances. Splits maintained the capacitive effect and metal fillets are pledged to the effect of inductance.

\section{Results and discussions}

There are plenty of ways to find out the effective parameters of a unit cell like NRW method, DRI, etc. This paper highlights the electromagnetic properties using the real values of $\epsilon, \mu$, and $\eta$ using $S_{11}$ and $S_{21}$.

\subsection{Analysis of the unit cell}

As the unit cell is fabricated on a Fr-4, which has an area of $81 \mathrm{~mm}^{2}$, it has been measured within a frequency range of 1 to $18 \mathrm{GHz}$. The simulation was done in the CST microwave studio and the result is compared with the measured one after the fabrication to measure the transmission coefficient $\left(\mathrm{S}_{21}\right)$. The measured result follows the similar pattern as there is a bit shitting of frequencies in the $\mathrm{C}$-band. The transmission coefficient exhibits a wide band with a coverage of L, C, X, and Ku-band. The first resonance is found in the L-band at frequency $1.63 \mathrm{GHz}$. Then a wide band from 
4.68 GHz to $17.18 \mathrm{GHz}$ with a little band gap of $500 \mathrm{MHz}$. The shifting is occurring due to fabrication error and the free space measurement process.

Figure 2(b) shows the current distribution of the unit cell at $6.50 \mathrm{GHz}$ and $13.5 \mathrm{GHz}$. In the proposed formation, inductances are formed by the metal strip and the capacitances are formed by the splits. A homogeneous wave with polarization is an incident in the $y$-axis and propagation in the $x$-axis to the structure. Additionally, dimensional scattering consequence, the electric field in the $\mathrm{x}$-direction induces a magnetic dipole in the y-direction, and the magnetic field in the y-direction induces an electric dipole in the x-direction [20]. Due to the antithetical geometry of the structure, a reverse current flow is noticed in the metal fillet of the configuration. Moreover, opposite current flows through the inner and outer surfaces of the resonator creates the stop band at this frequency [21].

Figure 3(a) shows the magnitude of the transmission coefficient $\left(S_{21}\right)$. By using $S_{21}$ and $S_{11}$ parameters, the effective parameters, i.e., effective permeability and effective permittivity can be obtained [22]. Figure 3(b) and (c) show the result of effective permittivity and effective permeability respectively.

To differentiate the effective permittivity $\left(\epsilon_{r}\right)$ and permeability $\left(\mu_{r}\right)$ with $\mathrm{S}_{11}$ and $\mathrm{S}_{21}$, the NRW method is applied.

$$
\begin{aligned}
& \epsilon_{r}=\frac{c}{j \pi f d} \times \frac{\left(1-V_{1}\right)}{\left(1+V_{1}\right)} \\
& \mu_{r}=\frac{c}{j \pi f d} \times \frac{1-V_{2}}{\left(1+V_{2}\right)}
\end{aligned}
$$

The effective refractive index $\left(\eta_{r}\right)$ can also be calculated from $S_{21}$ and $S_{11} \quad$ [2]:

$$
\eta_{r}=\frac{c}{j \pi f d} \times \sqrt{\frac{\left(S_{21}-1\right)^{2}-S_{11}^{2}}{\left(S_{21}+1\right)^{2}-S_{11}^{2}}}
$$

The magnetic dipole moment, which is created because the electric field is subjected to generate an artificial magnetism of the resonator. Eventually, that turns out to be an effective negative permeability. On the other hand, the magnetic resonance is superimposed to the corresponding electric resonance, which correlates to the effective negative permittivity. This overlapping turns out to be the effective negative refractive index of the composite medium.

Figure 3(b) shows negative permittivity at resonating points. It shows negativity at 2.60 to $5.16 \mathrm{GHz}, 6.63$ to 9.31 $\mathrm{GHz}$ and 13.03 to $16.18 \mathrm{GHz}$. Figure 3 (c) exhibits the negative permeability at 7.74 to $13.07 \mathrm{GHz}$ and 13.88 to $16.55 \mathrm{GHz}$. At lower frequencies, the current flow matches the applied

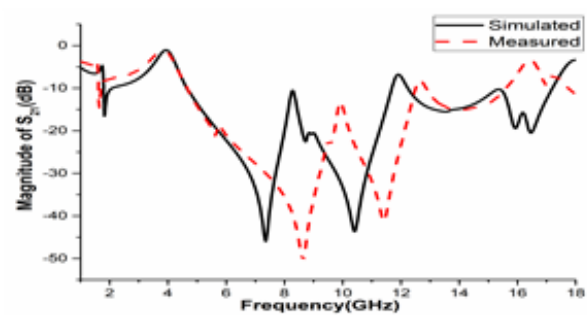

(a)

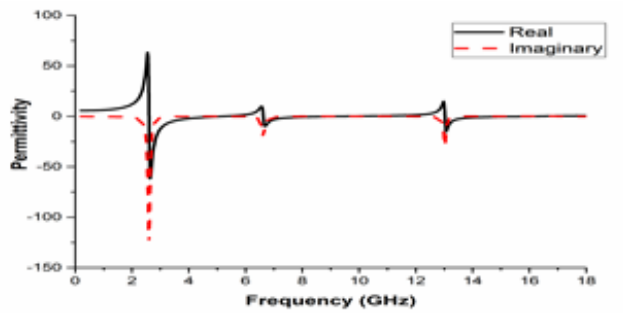

(b)

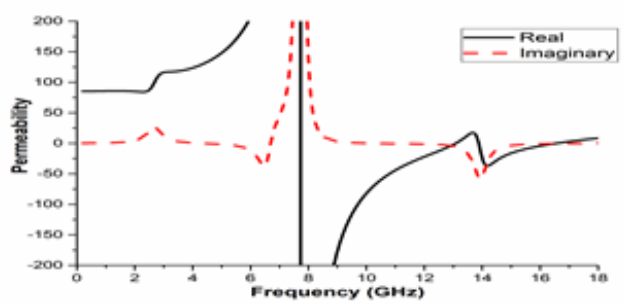

(c)

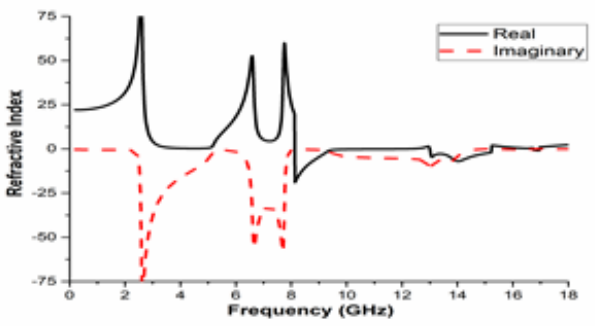

(d)

Figure 3: (a) Measured and simulated results of $S_{21}$;Real and imaginary values of (b) effective permittivity $(\epsilon)$ vs frequency; (c) effective permeability $(\mu)$ vs frequency; $(d)$ refractive index $(\eta)$ vs frequency

field. But in the case of higher frequencies, it is not possible for the current to cope up with the applied field when the permeability becomes negative. In the gap, there is a charge produced of a SRR, which is regulated to a fluctuating magnetic field. Both current and applied field remain in same phase at lower frequencies, but it fails to remain in phase in higher frequencies and as a result, negative permeability is produced.

In Figure 3(d), real and imaginary parts of $\eta$ are plotted as a function of frequency. The curve shows negativity at 8.13 to $12.14 \mathrm{GHz}, 13.01$ to $15.22 \mathrm{GHz}$ and 16.73 to $16.95 \mathrm{GHz}$. Table 2 shows the frequency range of refractive indices with effective parameters of the unit cell at different resonating frequency bands. The refractive index 


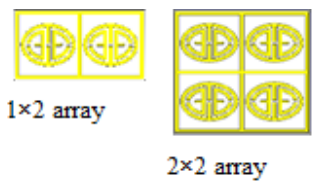

(a)

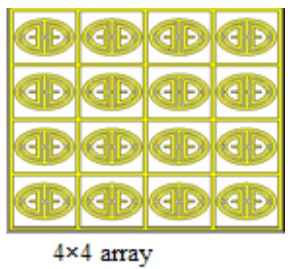

$-1 \times 2$
$-2 \times 2$
$-2 \times 2 \times 4$

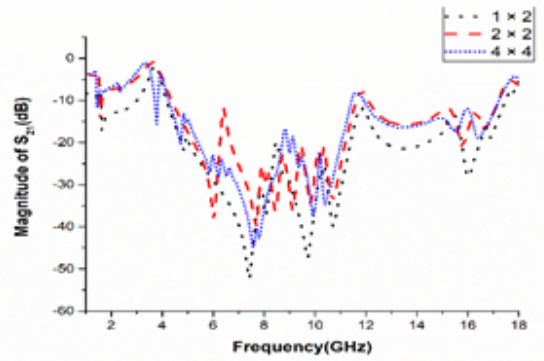

(b)
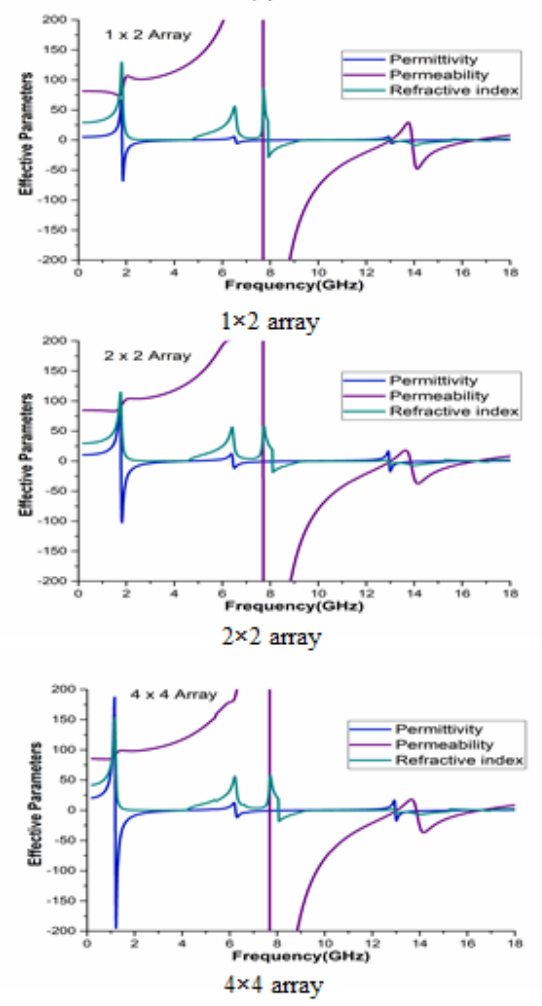

(c)

Figure 4: Unit structure (a) Different array formation; (b) $S_{21}$ vs frequency; (c) Effective parameters vs frequency for the $1 \times 2,2 \times 2$, $4 \times 4$ array.

shows negativity when the permittivity and permeability both become negative. Here $\eta$ shows certain negativity at different bands of frequencies. Hence, the designed unit cell has significant portions, where all the three effective parameters become negative. Therefore, this configuration can be considered as a double-negative metamaterial as it has negative peaks at $8.14 \mathrm{GHz}$ and $14.01 \mathrm{GHz}$ in all the
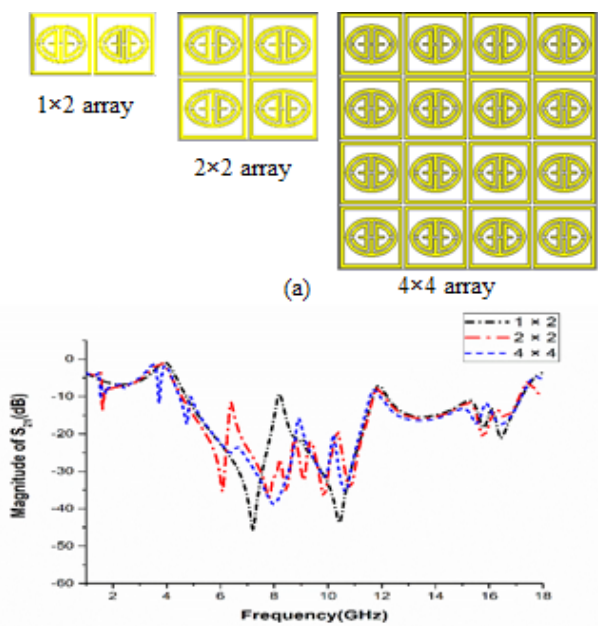

(b)
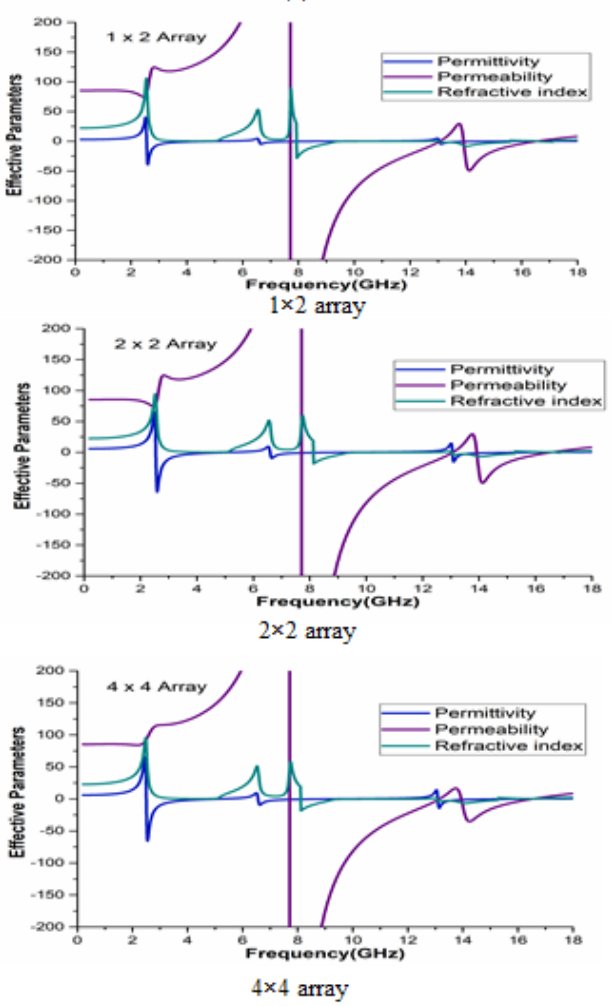

(c)

Figure 5: (a) Different array formation; (b) $S_{21}$ vs frequency; (c) Effective parameters vs frequency for the $1 \times 2,2 \times 2,4 \times 4$ array of the unit cell structure.

three effective parameters, which is shown in Table 2 with bandwidths.

\subsection{Array analysis}

Figure 4 describes the array formation of the unit structure and unit cell structure, which are placed horizontally for 1 
Table 2: Parameters of the unit cell

\begin{tabular}{cccc}
\hline Effective parameters & Frequency Range $(\mathrm{GHz})$ & Covered Bands & Values at 8.14 and $14.01 \mathrm{GHz}$ \\
\hline Permittivity $(\epsilon)$ & 2.60 to $5.16,6.63$ to $9.31 \& 13.03$ to $16.18 \mathrm{GHz}$ & $\mathrm{S}, \mathrm{C}, \mathrm{X} \& \mathrm{Ku}$ & $-0.56 \&-1.15$ \\
Permeability $(\mu)$ & 7.74 to $13.07 \& 13.88$ to $16.55 \mathrm{GHz}$ & C, $\mathrm{X} \& \mathrm{Ku}$ & $-292.49 \&-26.21$ \\
Refractive Index $(\eta)$ & $\mathbf{8 . 1 3}$ to $12.14,13.01$ to $15.22 \& 16.73$ to $16.95 \mathrm{GHz}$ & $\mathrm{X} \& \mathrm{Ku}$ & $-18.32 \&-6.99$ \\
\hline
\end{tabular}

$\times 2$ array and both vertically and horizontally on the basic unit structure for higher degrees of arrays on the same Fr-4 substrate. The array structure is measured within the frequency range of 1 to $18 \mathrm{GHz}$. For unit structure, both the patches are placed $0.5 \mathrm{~mm}$ apart from each other on the substrate. On the other hand, in the case of unit cell structure, the gap between the patches is $1 \mathrm{~mm}$ and the similar approach was used to assess the attainment of the array.

\subsubsection{Unit structure analysis}

Figure 4(a) shows array formation and (b) shows the transmission coefficient of the array structures. It is apparent that the resonances of the frequencies are found at the same points as the unit cell, but having higher negative magnitudes.

The S21 improves a bit as there is no band gap in between 4.68 to $17.18 \mathrm{GHz}$ in case of $1 \times 2$ array. But it shows a little gap of about $100 \mathrm{MHz}$ for $2 \times 2$ and $4 \times 4$ array. Figure 4(c) shows the real values of the permittivity, permeability and refractive index as a function of frequency of array structures.

From Figure 4(c), it is observed that the negative values for the single unit cell and the array structures are quite similar. The differences among them are the amplitudes or magnitudes and the resonating points shifted a bit to lower frequencies. The negative magnitude decreases in cases of permittivity. But in case of permeability, the negative magnitude increases at resonating points. In case of the refractive index, only the negative qualities are counted. The results of the array structures show similarity with the unit structure. All the effective parameters of these array structures are summarized in Table 3. All the arrays show double negative characteristics at 8.14 and $14.0 \mathrm{GHz}$.

\subsubsection{Unit cell structure analysis}

Figure 5 shows the design of unit cell structures of $1 \times 2$, $2 \times 2$ and $4 \times 4$ array. Here, the total unit cell is arranged horizontally for $1 \times 2$ array and for higher formations, the unit cells are placed $0.5 \mathrm{~mm}$ apart both vertically and horizontally, based on their degree. And the structures are operated at the frequency range of 1 to $18 \mathrm{GHz}$. The same procedure is followed to evaluate the unit cell and results, whichare compared with the array structures.

The array formations, effective parameters and the transmission coefficient of the unit cell structures are shown in Figure 5. Array formations are shown in Figure 5(a), S-parameter is shown in Figure 5(b) and Figure 5(c) contains the real values of effective parameters.

The demonstration was done between two square unit cells. They are actually two different working cells, but the output was quite identical to the single unit cell structure. And the same procedure is repeated for a higher degree of array formations. It is evident from the figure, at lower frequencies, there is a slight deviation in the effective parameters including transmission coefficient. The resonating points shifts a bit from the basic structure. In case of $\mathrm{S}_{21}$, the effect is a bit higher. There is no resonance in the Lband except $2 \times 2$ formation with a negligible spike. Moreover, instead of getting the double negative at $8.14 \mathrm{GHz}$, the point shifts to $7.94 \mathrm{GHz}$ to show the characteristic. But with the increase of frequencies, the unit cell structures showed good commitment to the basic unit cell. However, the unit cell still carries the double-negative characteristic at some extent.

The transmission coefficient of $13 \mathrm{GHz}$ with a $500 \mathrm{MHz}$ band gap in the middle is demonstrated for all of these configurations. The effective parameters of the resonators cover C, X, and Ku-band independently with double negative phenomena at $\mathrm{X}$ and $\mathrm{Ku}$-band, which is similar to basic unit cell. All the effective parameters of these unit cell structures are summarized in Table 4.

\section{Comparative analysis of the configurations}

In this paper, total observation is made on S-parameter, effective permittivity, effective permeability, and refractive index. In the proposed formation, inductances are formed by the metal strip and the capacitances are formed by the 
Table 3: Frequency range of effective parameters of array structures

\begin{tabular}{cccc}
\hline Effective parameters & Array Structures & Frequency Range $(\mathrm{GHz})$ & Covered Bands \\
\hline \multirow{3}{*}{ Permittivity $(\epsilon)$} & $1 \times 2$ & $1.83-4.76,6.55-9.19 \& 13.92-16.45$ & \\
& $2 \times 2$ & $1.78-4.61,6.44-9.19 \& 12.96-16.27$ & L, S, C, X \& Ku \\
& $4 \times 4$ & $1.19-4.22,6.27-9.12 \& 12.98-16.20$ & \\
Permeability $(\mu)$ & $1 \times 2$ & $7.71-13.01 \& 13.92-16.57$ & $\mathrm{C}, \mathrm{X} \& \mathrm{Ku}$ \\
& $2 \times 2$ & $7.71-13.01 \& 13.85-16.58$ & \\
Refractive Index $(\eta)$ & $4 \times 4$ & $7.69-13.03 \& 13.87-16.55$ & $\mathrm{C}, \mathrm{X} \& \mathrm{Ku}$ \\
& $1 \times 2$ & $7.92-12.32,12.98-15.56 \& 16.63-17.18$ & \\
\hline
\end{tabular}

Table 4: Frequency range of effective parameters for unit cell array structures

\begin{tabular}{cccc}
\hline Effective parameters & Array Structures & Frequency Range $(\mathrm{GHz})$ & Covered Bands \\
\hline \multirow{3}{*}{ Permittivity $(\epsilon)$} & $1 \times 2$ & $2.56-5.15,6.59-9.28 \& 13.05-16.41$ & \\
& $2 \times 2$ & $2.55-5.15,6.60-9.27 \& 13.06-16.16$ & $\mathrm{~S}, \mathrm{C}, \mathrm{X} \& \mathrm{Ku}$ \\
& $4 \times 4$ & $2.51-5.13,6.57-9.26 \& 13.10-16.09$ & \\
Permeability $(\mu)$ & $1 \times 2$ & $7.73-13.06 \& 13.92-16.55$ & \\
& $2 \times 2$ & $7.73-13.01 \& 13.92-16.54$ & $\mathrm{C}, \mathrm{X} \& \mathrm{Ku}$ \\
Refractive Index $(\eta)$ & $4 \times 4$ & $7.72-13.14 \& 13.96-16.52$ & \\
& $1 \times 2$ & $7.94-12.46,13.01-15.58 \& 16.61-17.12$ & \\
& $2 \times 2$ & $8.13-12.17,13.07-15.25 \& 16.77-16.86$ & $\mathrm{C}, \mathrm{X} \& \mathrm{Ku}$ \\
\hline
\end{tabular}

Table 5: Covered area and relative bandwidths by refractive index of different configurations for double negative characteristic

\begin{tabular}{ccccc}
\hline Structure & Frequency range $(\mathrm{GHz})$ & Band width & Covered Bands & Type of Metamaterial \\
\hline Unit Cell & $8.14-9.3113 .80-15.22$ & 1.171 .42 & $\mathrm{X} \& \mathrm{Ku}$ & DNG \\
$1 \times 2$ Structure & $7.92-9.1913 .92-15.56$ & 1.271 .64 & $\mathrm{X} \& \mathrm{Ku}$ & DNG \\
$1 \times 2$ Unit cell Structure & $7.94-9.2713 .92-15.58$ & 1.331 .66 & $\mathrm{X} \& \mathrm{Ku}$ & $\mathrm{DNG}$ \\
$2 \times 2$ Structure & $8.10-9.1913 .85-15.24$ & 1.091 .39 & $\mathrm{X} \& \mathrm{Ku}$ & DNG \\
$2 \times 2$ Unit cell Structure & $8.13-9.2713 .92-15.25$ & 1.141 .33 & $\mathrm{X} \& \mathrm{Ku}$ & DNG \\
$4 \times 4$ Structure & $8.06-9.1213 .87-15.24$ & 1.061 .37 & $\mathrm{X} \& \mathrm{Ku}$ & DNG \\
$4 \times 4$ Unit Cell Structure & $8.12-9.2613 .96-15.27$ & 1.141 .31 & $\mathrm{X} \& \mathrm{Ku}$ & DNG \\
\hline
\end{tabular}

Table 6: Comparison the proposed unit cell with the previous unit cell

\begin{tabular}{cccc}
\hline Previous Work & Dimensions $\left(\mathrm{mm}^{2}\right)$ & Resonance Frequency & Effective Medium Ratio \\
\hline & $10 \times 10$ & X-Band & 3.58 \\
Benosman et al., 2012 [16] & $3 \times 3$ & Ku and K-Band & 6.39 \\
Zhou et al., 2015 [17] & $15 \times 15$ & X and Ku-Band & 2.10 \\
Rizwan et al., 2014 [24] & $2 \times 2$ & K and Ka-Band & 6.30 \\
Malik et al., 2013 [25] & $25 \times 25$ & C and X-Band & 2.10 \\
Proposed Metamaterial & $9 \times 9$ & X and Ku-Band & 10.19 \\
\hline
\end{tabular}

splits. Electric resonances are produced by coupling between the gaps and electric fields, when the applied electromagnetic wave propagates along the structure. More- over, magnetic resonances are formed by the coupling between the magnetic fields and loops [21]. The splits of the proposed metamaterial structure as capacitor as they will 
be storing energy in terms of an electric field. Due to electric resonance and in fact this possesses a dielectric response that provides a permittivity. The following circular structure to store its energy primarily as a magnetic field. So it has a magnetic resonance that gives rise to permeability. The magnetic dipole moment, which is created because the electric field is subjected to generate an artificial magnetic field of the resonator. And eventually that turns out to be an effective negative permeability. On other the hand, the magnetic resonance is superimposed to the corresponding electric resonance, which is correlated to effective negative permittivity. This overlapping turns out to be effective negative refractive index of the composite medium. The negative properties of the permittivity and permeability has altered a little bit due to the polarization effect on the interior construction of the interconnected array structures [23]. Different array structures with different formations vary the capacitance which has a succinct impact on the coupling of the overall circuit and the change in polarization. As a result, the variation points out to the effective parameters by changing their negative properties a bit.

All the results have shown unique, but not contradictory information throughout the methodology. Based on the comparison of $1 \times 2,2 \times 2,4 \times 4$ arrays and $1 \times 2,2 \times 2,4$ $\times 4$ unit structure, it is found that the metamaterial shows double negativity at $\mathrm{X}$ and Ku-bands. It has covered 8.13 to $9.31 \mathrm{GHz}$ (bandwidth of $1.18 \mathrm{GHz}$ ) and 13.88 to 15.22 (bandwidth of $1.34 \mathrm{GHz}$ ) in basic unit structure. Among these set of results, 8.14 and $14.01 \mathrm{GHz}$ is the two frequencies where the double negative character of all sorts of configurations is found. Table 5 shows the covered area and relative bandwidths by the refractive index of different configurations for double negative characteristic.

From the Table 5, it is evident that all the configurations show similar double negative characteristic of the respective frequency range. But more stability is found among basic unit cell, $1 \times 2,2 \times 2$ and $4 \times 4$ array with higher bandwidths. Besides $1 \times 2,2 \times 2$ and $4 \times 4$ unit cell structures shown fluctuating results with less bandwidth with respect to other configurations.

In case of array analysis, all the unit structures show similar results, where unit cell structures show similarity among themselves. The reason behind the analysis of unit cell structures was to validate the metamaterial quality with the increase in gaps among themselves, as they have to be periodic in case of application. The overall inductance in a particular unit cell was fixed. The only scope was the gap that could change the potential values of the capacitances. The impact of coupling in the structure is shown due to different array formation. Although, all the formations follow the basic metamaterial characteristics and show similar results in every effective parameters.

Table 6 illustrates the comparisons of the frequency bands and the effective medium ratio of proposed design with previous work. The proposed structure demonstrates the effective medium ratio, which is more than 10 and is applicable for X-and Ku-band applications. Islam et al. [9], proposed a unit cell with a dimension of $10 \times 10 \mathrm{~mm}^{2}$, applied for invisibility cloaking but the EMR is $<4$. Benosman and Hacene [16] and Rizwan et al. [24] both introduced metamaterial structures of 3 and $2 \mathrm{~mm}$, respectively. Both of them have a common working range of frequency (Kband in common) with an EMR of around 6.30. Moreover, Mallik et al. [25] proposed meta-structures of $25 \mathrm{~mm}$, Zhou and Yang [17] of $15 \mathrm{~mm}$. All the mentioned studies with different dimensions are having different ranges of frequencies. But none of these researches have exceeded the EMR of the proposed metamaterial. However, this $9 \mathrm{~mm}$ Meta structure has a band coverage of $\mathrm{X}$ and Ku bands with an EMR of 10.19 .

\section{Conclusions}

This paper presents the framework of the modified Theta shaped unit cell and a correlation is contrived on a transmission coefficient, relative permeability, permittivity and refractive index. The analyses and the comparisons are made on unit cell, $1 \times 2,2 \times 2$ and $4 \times 4$ array structures with $1 \times 2,2 \times 2$ and $4 \times 4$ unit cell structures. The transmission coefficient $\left(\mathrm{S}_{21}\right)$ is calculated and compared with different array formations. The transmission coefficient covered L, C, X and Ku bands for all the configurations. Negative effective parameters are also found in all the structures. However, unit cell, $1 \times 2,2 \times 2$ and $4 \times 4$ array structures shown good agreement to the effective parameters. Even the negative values of each of the effective parameters are found on the $\mathrm{X}$ and $\mathrm{Ku}$ bands at 8.14 and $14.01 \mathrm{GHz}$ with a bandwidth of more than 1.20 and $1.32 \mathrm{GHz}$, respectively. It certainly represents the dual band double negative characteristic of the proposed compact design. Thus, these structures are valid for the application of dual bands and satellite communication. It can also be a promising choice for double negativity. The modified Theta shaped structure can be an auspicious alternative to new metamaterials, especially in utilizations where metamaterials are the only requirement. 
Acknowledgement: This work was supported by the Universiti Kebangsaan Malaysia, Research University Grant, Code: DPP-2018-004.

\section{References}

[1] Veselago V.G., The electrodynamics of substances with simultaneously negative values of $\epsilon$ and $\mu$, Sov. Phys., 1968, 10, 509 . 514.

[2] Smith D.R., Padilla W.J., Vier D.C., Nemat-Nasser S.C., Schultz S., Composite medium with simultaneously negative permeability and permittivity, Phys. Rev. Lett., 2000, 84, 4184-4187.

[3] Sultan K., Abdullah H., Abdallah E., E. Hashish, Low-SAR, Miniaturized printed antenna for mobile, ISM, and WLAN services, IEEE Ant.Wirel. Propag. Lett., 2013, 12, 1106-1109.

[4] Faruque M.R.I., Islam M.T., Misran N., Design analysis of new metamaterial for EM absorption reduction, Prog. Electromagn. Res., 2012, 124, 119-135.

[5] Islam M.M., Islam M.T., Samsuzzaman M., Faruque M.R.I., Compact metamaterial antenna for UWB applications, Electron. Lett., 2015, 51, 1222-1224.

[6] Khan O.M., Islam Z.U., Islam Q.U., Bhatti F.A., Multiband HighGain Printed Yagi Array Using Square Spiral Ring Metamaterial Structures for S-Band Applications, IEEE Ant. Wirel. Propag. Lett., 2014, 13, 1100-1103.

[7] Alam M.J., Faruque M.R.I., Islam M.T., Labyrinth double split open loop resonator based bandpass filter design for S, C and X-band application, J. Phys. D: Appl. Phys..2018, 51, 265102.

[8] Alam M.J., Faruque M.R.I., Islam M.T., Open Loop ResonatorBased Triple Passband Filter for $1.5 \mathrm{GHz}, 2.45 \mathrm{GHz}$ and $3.65 \mathrm{GHz}$ Applications. J. Elec. Mat., 2018, doi.org/10.1007/s11664-0186516-y.

[9] Islam S.S., Faruque M.R.I., Islam M.T., A Near Zero Refractive Index Metamaterial for Electromagnetic Invisibility Cloaking Operation, Materials, 2015, 8, 4790-4804.

[10] Landy N., Smith D.R., A full-parameter unidirectional metamaterial cloak for microwaves, Nat. Mater., 2013, 12, 25-28.

[11] Houshmand M., Zandi M.H., Gorji N.E., Modeling of Optical Losses in Perovskite Solar Cells, Sup.Latt. Micro., 2016, 97, 1, 424-42.

[12] Song K., Fu Q., Zhao X., U-Shaped multi-band negative-index bulk metamaterials with low loss at visible frequencies, Phys. Scr., 2011, 84, 035402.
[13] Huang Y., Wen J., Yang Y., Xie K., Tunable dual-band ferritebased metamaterials with dual negative refractions, Appl. Phys. A,2012, 106, 79-86.

[14] Hossain M.I., Faruque M.R.I., Islam M.T., Ullah M.H., A New Wide-Band Double-Negative Metamaterial for C- and S-B and Applications, Materials, 2015, 8, 57-71.

[15] Alam M.J., Faruque M.R.I., Hossain M.J., IslamM.T., Architecture of a unified split P-shaped swarming metamaterial for thermal mutation, Micro. Opt. Tech. Let., 2018, 60, 6, 1388-1395,.

[16] Benosman H., Hacene N.B., Design and Simulation of Double "S" Shaped Metamaterial, Int. J. Comput. Sci., 2012, 9, 534-537.

[17] Zhou Z., Yang H., Triple-Band asymmetric transmission of linear polarization with deformed S-shape bilayer chiral metamaterial, Appl. Phys. A, 2015, 119, 115-119.

[18] Alam M.J., Faruque M.R.I., Hossain M.J., Islam M.T., Depiction and analysis of a modified $\mathrm{H}$-shaped double-negative meta atom for satellite communication, Int. J. Micr. Wire. Tech., 2018, 1-11.

[19] Alu A., Bilotti F., Vegni L., Analysis of L-L transmission line metamaterials with coupled inductance, Micr. Opt. Tech. Let., 2006, 49, 94-97.

[20] Hasar U.C., Barroso J.J., Sabah C., Kaya Y., Ertugrul M., Stepwise technique for accurate and unique retrieval of electromagnetic properties of bianisotropic metamaterials, J. Opt. Soc. Amer. B - Opt. Phy., 2013, 30, 1058-1068.

[21] Hasan M., Faruque M.R.I., Islam S.S., A New Compact DoubleNegative Miniaturized Metamaterial for Wideband Operation, Materials, 2016, 9, 1-12.

[22] Luukkonen O., Maslovski S.I., Tretyakov S.A., A Stepwise Nicolson-Ross-Weir-Based Material Parameter Extraction Method. IEEE Ant.Wirel. Propag. Lett., 2011, 10,1295-1298.

[23] Hossain M.J., Faruque M.R.I., Islam M.T., Effective Medium Ratio Obeying Wideband Left-Handed Miniaturized Meta-atoms for Multi-band Applications, J. Electron. Mater., 2018, 47, 3,18591870.

[24] Rizwan M., Jin H.B., Rehman F., Hou Z.L., Li J.B., ButtF.K., AliZ., Dual-band tunable negative refractive index metamaterial with F-Shape structure, Centr. Europ. J. Phys.,2014, 12, 578-581.

[25] Mallik A., Kundu A.S., Goni M.O., Design of a novel two rectangular U-shaped double negative metamaterial. Proceedings of the 2013 International Conference on Informatics, Electronics \& Vision (ICIEV), Dhaka, Bangladesh, 2013, 17-18 May. 